\title{
LEVELS OF PROINFLAMMATORY CYTOKINES IL-17 AND IL-23 IN PATIENTS WITH ALZHEIMER'S DISEASE, MILD COGNITIVE IMPAIRMENT AND VASCULAR DEMENTIA
}

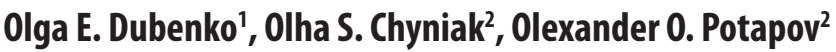 \\ ${ }^{1} K$ HARKIV MEDICAL ACADEMY OF POSTGRADUATE EDUCATION, KHARKIV, UKRAINE \\ 2SUMY STATE UNIVERSITY, SUMY, UKRAINE
}

\begin{abstract}
The aim: To research differences of interleukin (IL)-17 and IL-23 serum levels in patients with Alzheimer's disease, vascular dementia and mild cognitive impairment. Material and methods: Serum levels of IL-17 and IL-23 were measure by ELISA for 15 patients with Alzheimer's disease, 14 with vascular dementia, 30 with mild cognitive impairment and 30 control individuals without cognitive impairment.

Results: Serum concentrations of IL-17 were significantly higher in Alzheimer's disease patients ( $P=0.0023)$ than control, in vascular dementia no significant differences $(P=0.4154)$. Level of IL-23 was significantly higher than control in Alzheimer's disease patients $(P=0.0170)$ and vascular dementia $(P=0.0002)$, but in Alzheimer's disease it was in 12.5 time higher. In total mild cognitive impairment patients no significant differences in interleukin concentration with control, but significant differences observed for amnestic form in IL-17 ( $\mathrm{P}=0.0436)$ and IL-23 ( $\mathrm{P}=0.0019)$.

Conclusions: IL-17 and IL-23 level significant higher in Alzheimer's disease patients compared with control and vascular dementia. From mild cognitive impairment levels of detectable interleukins was higher in amnestic form that may be early marker of progression in Alzheimer's disease.
\end{abstract}

KEY WORDS: Alzheimer's disease, vascular dementia, interleukins

Wiad Lek. 2021;74(1):68-71

\section{INTRODUCTION}

Among old-related dementia, Alzheimer's disease $(\mathrm{AD})$ is the most common and characterized by a progressive and irreversible deterioration of cognitive and function abilities [1]. Dementia was name major neurocognitive disorder (NCD) in the fifth edition of the Diagnostic and Statistical Manual of Mental Disorders (DSM-5) [2]. Mild NCD is a diagnostic category in DSM-5 added to recognize the substantial clinical need of individuals living with this disorder, which might also be termed mild cognitive impairment (MCI). Mild NCD possible is preddementia stage in $\mathrm{AD}$ but not always a precursor of major NCD. AD is a multifactorial etiopathogenesis disorder and neuroinflammatory processes are a central feature in which microglia are over-activated, resulting in increased production of pro-inflammatory cytokines. Evidence suggests that different cytokines, including interleukins (IL) IL-6, IL10 , IL-12, TNF- $\alpha$ and TGF- $\beta$ are actively participated in AD pathogenesis [3]. IL-17 and IL-23 augmented in AD patients upon stimulating of cell with $A \beta$ in vitro and play role in $\mathrm{AD}$-associated neuroinflammation [4].

\section{THE AIM}

The aim of this study to research differences of interleukin (IL)-17 and IL-23 serum levels in patients with Alzheimer's disease, vascular dementia and mild cognitive impairment.

\section{MATERIALS AND METHODS}

The study involved 59 patients with cognitive impairment (43 men and 46 women, average age $-66.8 \pm 8.4$ years), of which 29 has major NCD and 30 mild NCD. $15(25.4 \%)$ patients with major NCD meet to updated criteria for clinical practice proposed for the diagnosis of Alzheimer's disease at the Alzheimer's Association of the National Institute of Aging [5, 6], 14 (23.7\%) - meet to criteria probable vascular dementia (VD) according to the NINDS-AIREN [7]. 30 patients with mild NCD was divide to amnestic MCI (aMCI) - 9 (15.25\%) patients if they had impairment in the memory domein and nonamnestic MCI (naMCI) - 21 (35.59\%) if they had impairment in any 1 or more of the nonmemory cognitive domain. There is no patients with early-onset dementia or MCI or family history of AD.

Inclusion criteria were: the objective confirmation of cognitive impairment according to clinical and neuropsychological tests based on criteria of propable $\mathrm{AD}$, probable VD and MCI, presence signs of cerebrovascular and neurodegenerative brain damage according to clinical and neuroimaging methods. Exclusion criteria were: severe somatic diseases, other mental disorders, traumatic brain damage and brain tumors, infections, epilepsy, Parkinson's disease, demyelinating and inherited degenerative diseases, alcohol consumption, intake of drugs that reduce 
Table I. Comparison of characteristics between patients with $A D, V D$ and $M C l$

\begin{tabular}{ccccccc}
\hline characteristics & $\begin{array}{c}\text { Major } \mathbf{N C D} \\
(\mathbf{n}=\mathbf{2 9 )}\end{array}$ & $\begin{array}{c}\text { Mild } \mathbf{N C D}(\mathbf{M C l}) \\
(\mathbf{n}=\mathbf{3 0})\end{array}$ & p value & AD $(\mathbf{n = 1 5 )}$ & VD (n=14) & p value \\
\hline Mean age (y) & $67.5 \pm 0.6$ & $65.6 \pm 0.8$ & 0.0638 & $67.9 \pm 0.8$ & $67.0 \pm 0.3$ & 0.3145 \\
\hline Male/female & $14 / 15$ & $18 / 12$ & 0.1640 & $5 / 10$ & $9 / 5$ & 0.0960 \\
\hline Arterial hypertension & 20 & 17 & 0.3290 & 6 & 14 & $<0.0001$ \\
\hline Smoking & 19 & 11 & 0.0270 & 5 & 14 & $<0.0001$ \\
\hline Diabetes mellitus & 13 & 2 & 0.0001 & 3 & 10 & 0.0050 \\
\hline Ischemic heart disease & 22 & 5 & $<0.0001$ & 8 & 14 & 0.0030 \\
\hline $\begin{array}{c}\text { Acute ischemic events } \\
\text { in anamnesis }\end{array}$ & 14 & 5 & 0.0090 & 0 & 14 & $<0.0001$ \\
\hline Mean MMSE score & $20.2 \pm 1.64$ & $25.2 \pm 0.85$ & 0.0083 & $18.8 \pm 0.56$ & $21.7 \pm 0.69$ & 0.0028 \\
\hline Mean MoCA test & $18.1 \pm 1.67$ & $24.2 \pm 0.86$ & 0.0018 & $16.6 \pm 0.50$ & $19.7 \pm 0.61$ & 0.0005 \\
\hline Mean FAB test & $11.7 \pm 0.77$ & $14.3 \pm 0,9$ & 0.0327 & $12.4 \pm 0.50$ & $11.1 \pm 0.36$ & 0.0468 \\
\hline Mean HIS score & $5.6 \pm 1.58$ & - & - & $2.4 \pm 0.50$ & $9.1 \pm 0.77$ & $<0.0001$ \\
\hline
\end{tabular}

cognitive function, taking corticosteroids, severe poststroke deficite, inability to have sufficient verbal contact.

For record vascular risk factors the patient's medical history and medication use was obtain. Hypertension was define by casual blood pressure $\geq 140 / 90 \mathrm{mmHg}$ or current use of antihypertensive drugs, diabetes was define by fasting glucose $\geq 7 \mathrm{mmol} / \mathrm{l}$ or use glucose-lowering agents.

The control group consisted 30 subjects (mean age 65.7 \pm 0.9 ) without cognitive deficit and serious illnesses. No significant differences observed for age, gender, education level between patients groups and control subjects.

All patients were examine to a comprehensive neuropsychological examination using the following tests and scales: Mini-Mental State Examination (MMSE), Montreal Cognitive Assessment (MoCA), Frontal Assessment Battery (FAB), Hachinski's Ischemic Scale - (HIS). The severity of cognitive impairment determined by the Clinical Dementia Rating (CDR). In addition, all patients evaluated using Magnetic Resonance Imaging (MRI).

Serum levels of cytokines of IL-17 and IL-23 was assayed using sandwich ELISA on "Chem Well 2900" immunoanalyzer (Awareness Technology, USA). Test systems using Bender Medsystems, Australia (IL-17 and IL-23) were assay in according with the manufactures instructions.

The work performed in accordance with the principles of the World Health Association Helsinki Declaration "Ethical Principles of Medical Research with Human Involvement as Object of Study" Order of the Ministry of Health of Ukraine No. 690 (dated September 23, 2009). Before inclusion in the study, patients and their relatives were inform with the study protocol and signed voluntary informed consent.

The IBM Statistical Package was use to perform statistical analyses. The level of significance was defined as $p<0.05$. $\chi^{2}$ test were conducted to compare clinical characteristics and Kruskal-Wallis test was applied to compare the concentration of the IL between different group.

\section{RESULTS}

In our study the vascular risk factors that associated with cognitive impairment was higher in patients with major NCD compared with mild NCD. However, in group with VD the incidence of arterial hypertension, smoking, congestive heart failure, diabetes mellitus and anamnesis of acute ischemic events was significantly higher compared with patients with $\mathrm{AD}$ (table 1).

The mean scores of MMSE and MoCA test was significantly lower in patients with AD comparable with VD $(\mathrm{p}=0.0028 ; \mathrm{p}=0.0005)$, particular in subtest orientation (3.4 \pm 0.51 vs $4.8 \pm 0.34, \mathrm{p}=0.0226)$, delayed recall $(1.8 \pm 0.4$ vs $2.9 \pm 0.2, \mathrm{p}=0.0108)$. Mean HIS score was higher in VD patients.

The detectable serum levels of IL-17 and IL-23 in patients with major NCD, AD and VD presented in table 2.

Levels of detectable interleukins were significantly higher in patients with $\mathrm{AD}$ comparable with $\mathrm{VD}(\mathrm{P}=0.0481)$. IL17 level was in 10 time higher in $\mathrm{AD}$ patients comparable control $(p=0.0023)$. In patients with VD no significant differences with control ( $\mathrm{p}=0.4154)$, but individual values in patients with VD was significantly greater than normal.

IL-23 level also was significant higher in $\mathrm{AD}$ patients than in control group $(\mathrm{p}=0.0170)$ and significant differences observed between patients with $\mathrm{AD}$ and $\mathrm{VD}(\mathrm{p}=0.0027)$. Level of IL-23 was in 42 time higher comparable with control and in 12.5 time higher comparable with VD patients. This result confirm that elevate concentration of IL-17 and IL-23 high specific for AD.

When comparison the IL-17 and IL-23 concentration in patients with total mild NCD and control no significant differences were found $(\mathrm{p}=0.1215 ; \mathrm{p}=0.4733)$ (table 3 ). However when compared patients with aMCI and naMCI significant differences found in IL-17 between aMCI and control ( $\mathrm{p}=0.0436)$.

No significant differences in serum concentration of IL23 observed in total mild NCD patients and control, but significant differences was found between aMCI patients 
Table II. Serum levels of the IL-17 and IL-23 in patients with AD, VD and control

\begin{tabular}{|c|c|c|c|c|c|}
\hline $\begin{array}{c}\text { Interleukin } \\
\text { concentration, pg/ml } \\
\text { Mean } \pm \text { SD }\end{array}$ & $\begin{array}{c}\text { Total major NCD } \\
\mathbf{n}=\mathbf{2 9}\end{array}$ & $\begin{array}{c}A D \\
n=15\end{array}$ & $\begin{array}{c}\text { VD } \\
n=14\end{array}$ & $\begin{array}{c}\text { Control } \\
n=30\end{array}$ & P value \\
\hline IL-17 & $13.11 \pm 5.11$ & $22.44 \pm 8.92$ & $3.11 \pm 1.35$ & $2.10 \pm 0.56$ & $\begin{array}{l}P_{1}=0.0335 \\
P_{2}=0.0023 \\
P_{3}=0.4154 \\
P_{4}=0.0481\end{array}$ \\
\hline IL-23 & $35.75 \pm 15.2$ & $64.33 \pm 22.41$ & $5.14 \pm 1.62$ & $1.53 \pm 0.20$ & $\begin{array}{l}P_{1}=0.0265 \\
P_{2}=0.0170 \\
P_{3}=0.0002 \\
P_{4}=0.0027\end{array}$ \\
\hline
\end{tabular}

$P_{1}$ - differences between major NCD and control

$P_{2}^{1}$ - differences between $A D$ and control

$\mathrm{P}_{3}^{2}$ - differences between VD and control

$\mathrm{P}_{4}$ - differences between $A D$ and VD

Table III. Serum levels of the IL-17 and IL-23 in patients with mild NCD and control

\begin{tabular}{|c|c|c|c|c|c|}
\hline $\begin{array}{c}\text { Interleukin } \\
\text { concentration, pg/ml } \\
\text { Mean } \pm \text { SD }\end{array}$ & $\begin{array}{l}\text { Total mild NCD } \\
\qquad \mathbf{N}=\mathbf{3 0}\end{array}$ & $\begin{array}{l}\mathrm{aMCl} \\
\mathrm{n}=9\end{array}$ & $\begin{array}{c}\text { naMCI } \\
n=21\end{array}$ & $\begin{array}{c}\text { Control } \\
n=30\end{array}$ & P value \\
\hline IL-17 & $4.04 \pm 1.10$ & $4.36 \pm 0.61$ & $3.90 \pm 0.58$ & $2.10 \pm 0.56$ & $\begin{array}{l}P_{1}=0.1215 \\
P_{2}=0.0436 \\
P_{3}=0.0344 \\
P_{4}=0.6411\end{array}$ \\
\hline IL-23 & $1.84 \pm 0.38$ & $2.80 \pm 0.17$ & $1.43 \pm 0.21$ & $1.53 \pm 0.20$ & $\begin{array}{l}P_{1}=0.4733 \\
P_{2}=0.0019 \\
P_{3}=0.7376 \\
P_{4}=0.0004\end{array}$ \\
\hline
\end{tabular}

$P_{1}$ - differences between mild NCD and control

$\mathrm{P}_{2}$ - differences aMCl and control

$\mathrm{P}_{3}^{2}$ - differences naMCl and control

$\mathrm{P}_{4}^{3}$ - differences between $\mathrm{aMCl}$ and naMCI

and control $(\mathrm{p}=0.0019)$ and $\mathrm{aMCI}$ and naMCI patients groups ( $\mathrm{p}=0.0004)$. Concentration of IL-23 was significantly higher in patients with aMCI compare with naMCI $(p=0.0004)$. Such differences confirm that aMCI may be early stage of $\mathrm{AD}$ and elevation of serum concentration IL-17 and IL-23 in patients may be addition markers of risk progression $\mathrm{aMCI}$ in $\mathrm{AD}$.

\section{DISCUSSION}

Alzheimer's disease $(\mathrm{AD})$ is a neurodegenerative disorder that is the most common cause of dementia. AD is characterize by two core pathologies, the presence of $\beta$-amyloid $(\mathrm{A} \beta)$ plaques and neurofibrillary tangles (NFTs). A number of investigations initially demonstrated that in addition to $\mathrm{A} \beta$ plaques and NFT, the brains of patients with $\mathrm{AD}$ exhibited evidence of a sustained inflammatory response [8]. This chronic neuroinflammation is attributed to activated microglia cells and the release of numerous cytokines. Many studies now point to the involvement of neuroinflammation playing a fundamental role in the progression of the neuropathological changes that are observe in $\operatorname{AD}[9,10]$. Such overproduction of IL-6 leads to chronic neuroinflammation and neurodegeneration [11]. IL-1 is a proinflammatory cytokine that is upregulated early in $\mathrm{AD}$ development and are considere crucial for $\beta$-amyloid plaque deposition. IL- $1 \beta$ is similarly elevated in both MCI and AD patients compared with controls, suggesting that increased IL- $1 \beta$ production begins early and remains elevated as the disease progresses. Specific IL- $1 \beta$ polymorphisms resulting in higher IL- $1 \beta$ production are linked to increased AD risk [12]. The participant of IL-10 that play anti-inflammatory and neuroprotective role in nervous system also investigated in $\mathrm{AD}$ [13]. The role of IL-17 and IL-23 is less elucidate. Research demonstrated that IL-23/T17 axis plays a role in AD-associated neuroinflammation and IL-17 in the production of Th17 [14]. In vitro studies suggest that IL-23 might promote Th17 development, stimulate Th17 expansion and prolong IL-17 production [15]. In previous study are observe the elevation of IL-18, IL-23 and IL-17 levels in Chinese patients with $\mathrm{AD}$ and differences between males and females [16]. In this study, we compared serum level IL-17 and IL-23 in patients with clinical diagnosis $\mathrm{AD}$ and $\mathrm{VD}$. Our results suggest that in $\mathrm{AD}$ patients interleukins significantly increase that reflect increase of inflammatory response, which could contribute to the development of neurodegeneration in $\mathrm{AD}$. 
Patients with aMCI are consider to be at high risk for $\mathrm{AD}$ [17]. Routine use of biomarkers such cerebrospinal fluid $A \beta_{1-42}$ still obstacle for identify the disease etiology [18] and search new biomarkers for it identify and early therapeutic intervention is important aim. In our study IL-17 and IL-23 was statistically significant higher in aMCI patients comparable with control group.

\section{CONCLUSIONS}

IL-17 and IL-23 level significant higher in Alzheimer's disease patients compared with control and vascular dementia. Levels of detectable interleukins was higher in aMCI compared with control and significant differences between aMCI and naMCI groups was demonstrate for IL-23. Future investigation may be elucidate a potential role of this interleukins as additional biomarkers for early predict of progression aMCI in Alzheimer's disease.

\section{REFERENCES}

1. Prince M., Bryce R., Albance E., Wimo A. et al. The global prevalence of dementia: a systematic review and metaanalysis. Alzheimers Dement. 2013; 9:63-75.

2. Salvadori E., Poggesi A., Pracucci G., Chiti A. et al. Application of the DSM-5 Criteria for Major Neurocognitive Disorder to Vascular MCI Patients. Dement Geriatr Cogn Disord 2018; 8:104-116.

3. SwardfagerW., LanctotK., Rothenburg L., Wong A. et al. A meta-analysis of cytokines in Alzheimer's disease. Biol Psychiatry 2010; 68:930-941

4. Vom Bern J., Prokop S., Miller K.R. et al. Inhibition of IL-12/23 signaling reduces Alzheimer's disease-like pathology and cognitive decline. Nat Med. 2012; 18:1812-1819.

5. McKhaann G.M., Chertkow H. et al. The diagnosis of dementia due to Alzheimer's disease: recommendations from the National Institue on Aging- Alzheimer's Association workgroups on diagnostic guidelines for Alzheimer's disease. Alzheimers Dement. 2011; 7:263-269.

6. Cummings J. Alzheimer's disease diagnostic criteria: practical applications. Alzheimer's Research \& Therapy. 2012; 4:35-40.

7. Sachdev P., Kalaria R., O'Brien J. et al. Diagnostic criteria for vascular cognitive disorders: a VASCOG statement. Alzheimer Dis Assoc Disord. 2014; 28: 206-218.

8. Ferreti M.T., Bruno M.A., Ducatenzeiler A., Klein W.L. et al. Intracellular A $\beta$-oligomers and early inflammation in a model of Alzheimer's disease. Neurobiol Aging. 2012; 33: 1329-1342.

9. Kinney J.W., Bemiller S.M., Murtishaw A.S., Leisgang A.M. et al. Inflammation as a central mechanism in Alzheimer's disease. Alzheimer Dement (NY). 2018; 4: 575-590.

10. Su F., Bai F., Zhang Z. Inflammatory Cytokines and Alzheimer's Disease:A Review from the Perspective of Genetic Polymorphisms. Neurosci. Bull. October, 2016; 32(5):469-480. DOI 10.1007/s12264-016-0055-4.

11. Dursun E., Gezen-Ak D., Hanağası H., Bilgiç B. et al. The interleukin 1 alpha, interleukin 1 beta, interleukin 6 and alpha-2-macroglobulin serum levels in patients with early or late onset Alzheimer's disease, mild cognitive impairment or Parkinson"s disease. J Neuroimmunol. 2015; 283:50-57.
12. Forlenza 0.V., Diniz B.S., Talib L.L., Mendonça V.A. et al. Increased serum IL-1beta level in Alzheimer's disease and mild cognitive impairment. Demen Geriatr Cogn Disord. 2009; 28:507-512.

13. D'Anna L., Abu-Rumeileh S., Fabris M., Pistis C. et al. Serum Interleukin-10 Levels Correlate with Cerebrospinal Fluid Amyloid Beta Deposition in Alzheimer Disease Patients. Neuro-Degenerative Dis. 2017;17:227-234.

14. Saresella M., Calabrese E., Marventano I. et al. Increased activity ofTh-17 and Th-9 lymphocytes and a skewing of the post-thymic differentiation pathway are seen in Alzheimer's disease. Brain Behav Immun. 2011; 25:539-547.

15. Aggarwal S., Ghilardi N., Xie M.H., de Sauvage F.J. et al. Interleukin-23 promotes a distinct CD4 cellactivation state characterized by the production if interleukin-17. J Biol Chem. 2003; 278:1910-1914.

16. Chen J.M., Jiang G.X., Li Q.W., Zhou Z.M. et al. Increased serum levels of interleukin-18, -23 and -17 in Chinese patients with Alzheimer's disease. Dement Geriatr Cogn Disord. 2014; 38: 321-329.

17. Shin S., Kim J.H., Cho J.H., Kim G.S. et al. Mild cognitive impairment due to Alzheimer Disease is less likely under the age of 65 . Alzheimer Dis Assoc Disord. 2015; 29:26-31.

18. Tabaraud F., Leman J.P., Milor A.M. et al. Alzheimer CSF biomarkers in routine clinical setting. Acta Neurol Scand. 2012; 125:416-423.

This study fulfilled according to scientific research of department of neurology and neurosurgery Kharkiv medical academy of postgraduate education "Diagnosis, fluence and outcomes of acute and chronic cerebrovascular disorders according to the results of clinic and paraclinic study" №0115U000143.

\section{ORCID and contributionship:}

Olga E. Dubenko: 0000-0002-4911-5613 A,D,F

Olha S. Chyniak: 0000-0002-6008-1039 ${ }^{B, C, D}$

Olexander O. Potapov: 0000-0002-9683-3685 ${ }^{B, E}$

\section{Conflict of interest:}

The Authors declare no conflict of interest.

\section{CORRESPONDING AUTHOR Olga E. Dubenko \\ Kharkiv medical academy of postgraduate education \\ 58 Amosova st., 61176 Kharkiv, Ukraine \\ tel:+30 0506601442 \\ e-mail:olgadubenk005@gmail.com}

Received: 05.03 .2020

Accepted: 14.10 .2020
A - Work concept and design, B - Data collection and analysis, C - Responsibility for statistical analysis, D -Writing the article, $\mathbf{E}$-Critical review, $\mathbf{F}$ - Final approval of the article 